TITLE:

\title{
The Effect of Monoglyceride Polymorphism on Cold-Flow Properties of Biodiesel Model Fuel
}

\section{$\operatorname{AUTHOR}(\mathrm{S})$ :}

Sugami, Yuitsu; Yoshidomi, Shinichiro; Minami, Eiji; Shisa, Noriko; Hayashi, Hitoshi; Saka, Shiro

\section{CITATION:}

Sugami, Yuitsu ... [et al]. The Effect of Monoglyceride Polymorphism on Cold-Flow Properties of Biodiesel Model Fuel. Journal of the American Oil Chemists' Society 2017, 94(8): 1095-1100

\section{ISSUE DATE:}

2017-08

URL:

http://hdl.handle.net/2433/250160

\section{RIGHT:}

This is the peer reviewed version of the following article: Sugami, Y., Yoshidomi, S., Minami, E., Shisa, N., Hayashi, H. and Saka, S. (2017), The Effect of Monoglyceride Polymorphism on Cold - Flow Properties of Biodiesel Model Fuel. J Am Oil Chem Soc, 94: 1095-1100, which has been published in final form at https://doi.org/10.1007/s11746-017-3016-9. This article may be used for non-commercial purposes in accordance with Wiley Terms and Conditions for Use of SelfArchived Versions: This is not the published version. Please cite only the published version.; この論文は出版社版であり ません。引用の際には出版社版をご確認ご利用ください。 
Authors: Yuitsu Sugami ${ }^{1} \cdot$ Shinichiro Yoshidomi ${ }^{1} \cdot$ Eiji Minami $^{1} \cdot$ Noriko Shisa $^{2}$.

Hitoshi Hayashi $^{2} \cdot$ Shiro Saka ${ }^{1}$

Title: The effect of monoglyceride polymorphism on cold-flow properties of biodiesel model fuel

\section{Affiliations and addresses:}

${ }^{1}$ Graduate School of Energy Science, Kyoto University, Yoshida-honmachi, Sakyo-ku, Kyoto 606-8501, Japan

${ }^{2}$ Material Engineering Division, Toyota Motor Corporation, Toyota-cho, Toyota, Aichi 471-8572, Japan

Corresponding author: Shiro Saka

E-mail: saka@energy.kyoto-u.ac.jp. Phone/Fax: +81-75-753-4738. 
Abstract: The cloud point of biodiesel refers to the temperature at which crystallization begins on cooling. However, solid precipitates are often formed at a temperature higher than cloud point during storage. Such precipitates are known to consist largely of monoglycerides (MGs) as intermediate compounds. MGs has high melting point, which are detrimental to the cold-flow properties of biodiesel. MGs have several polymorphic forms, including $\alpha, \beta^{\prime}$ and $\beta$, with different melting points ( $\alpha$ $<\beta^{\prime}<\beta$ ), and this fact makes the behavior of biodiesel at low-temperature complicated. In this study, the precipitation behavior of MGs in biodiesel is discussed focusing on polymorphism using 1-monopalmitin and methyl oleate mixtures as a model biodiesel fuel. The cloud points (CP) measured were close to the calculated solid-liquid equilibrium curve for $\alpha$-type 1-monopalmitin. However, precipitates formed at temperatures higher than the $\mathrm{CP}$ when the mixtures were held at temperatures lower than the equilibrium curve of the $\beta^{\prime}$ form. This indicates that the $\beta^{\prime}$ form causes a risk of precipitation at temperatures above the $\mathrm{CP}$ and that the $\mathrm{CP}$ would not be a suitable indicator of the cold-flow properties of biodiesel.

Keywords: Biodiesel $\cdot$ Monoglyceride $\cdot$ Polymorphism $\cdot$ Cloud point 


\section{Introduction}

Fatty acid methyl esters (FAMEs) are produced from plant oils and are the main component of biodiesel, which is widely used as an alternative to petroleum diesel with minor or no engine modification required. FAMEs are a renewable and carbon neutral fuel that do not increase net $\mathrm{CO}_{2}$ release to the atmosphere, and these advantages may mitigate environmental problems such as exhaustion of fossil resources and global warming.

Specification standards for biodiesel have been established in many countries with the spread of biodiesel use to ensure quality. Nevertheless, some problems occur in practical use of biodiesel owing to corrosion of rubber [1], poor oxidation stability [2] and poor cold-flow properties [3, 4]. Poor cold-flow properties especially restrict the use of biodiesel in cold climates and during the winter season.

There are three kinds of cold-flow properties for liquid fuels: cloud point (CP), cold filter plugging point (CFPP), and pour point (PP). The CP refers to the temperature at which a cloud of wax crystals first appears in a liquid when it is cooled [5]. The CFPP is the lowest temperature where a given volume $(20 \mathrm{~mL})$ of a fuel successfully passes through a wire mesh $(45 \mu \mathrm{m})$ within $60 \mathrm{sec}$. The PP refers to the lowest temperature 
where a fuel shows fluidity when it is tipped. The CP is a useful indicator for the formation of precipitates because it generally has the highest value among these properties. However, precipitates are often found at a temperature higher than the $\mathrm{CP}$, and the precipitates consist mainly of saturated monoglycerides (MGs) [6], which are intermediate compounds from the transesterification reaction used to produce biodiesel that slightly contaminate the fuel.

MGs precipitate easily because they have higher melting points than the corresponding methyl esters. MGs have several polymorphic crystalline forms referred to as sub- $\alpha, \alpha, \beta^{\prime}$ and $\beta$. Each form has a different melting point (sub- $\alpha<\alpha<\beta^{\prime}<\beta$ ) [7-9], and the polymorphs of MGs make the cold-flow properties of biodiesel complicated. A pure liquid MG crystallizes first as the $\alpha$ or sub- $\alpha$ form when cooled [10], and these forms can interconvert reversibly. The $\alpha$ and sub- $\alpha$ forms are metastable and irreversibly convert to the $\beta^{\prime}$ form, which finally changes to the most stable $\beta$ structure after a certain transition time [10]. However, the behavior of MG polymorphs in FAMEs remains unclear. Although some studies reported that the effects of MGs in biodiesel are significant on the cold-flow properties [6, 11, 12], it seems that the mechanism of precipitation has not been established. 
In our another study [13], we conducted a thermodynamic study for mixtures of

MGs and FAMEs to predict the solid-liquid equilibrium, and found that binary mixtures

of MGs and FAMEs behave as non-ideal solutions, but the temperature of solid-liquid equilibrium is well predicted by a modified universal quasi-chemical functional-group activity coefficients (UNIFAC) model, referred to as "modified UNIFAC (Dortmund)" [14]. In the current study, the precipitation behavior of MGs in FAMEs is discussed based on this prediction model to reveal the reason for inconsistencies between the CP and practical precipitation temperature, focusing on the effect of MG polymorphism.

\section{Materials and Methods}

\section{Materials}

In typical plant oils, palmitic (C16:0), stearic (C18:0), oleic (C18:1), linoleic (C18:2) and linolenic (C18:3) acids are the major fatty acid moieties; the first and second numbers in parentheses mean the number of carbon atoms and double bonds, respectively. The MGs of palmitic acid are probably an important cause of precipitation because they have higher melting points than unsaturated MGs. We chose a binary mixture of 1-monopalmitin and methyl oleate as a model for biodiesel. 1-Monopalmitin (C16:0 MG, 
98\%) was purchased from Tokyo Chemical Industry Co., Ltd., Tokyo, Japan, and methyl

oleate (C18:1 FAME, >99\%) was obtained from Sigma-Aldrich Japan Co., LLC., Tokyo,

Japan. These chemicals were used without purification. Binary mixtures were prepared by adding 1-monopalmitin to methyl oleate in concentrations of $0.10,0.50$ and $1.0 \mathrm{wt} \%$.

\section{Experimental and Analytical Methods}

CPs of the binary mixtures prepared were measured by an automated cloud point tester (APM-7, Tanaka Scientific Co. Ltd., Tokyo, Japan) in conformity to ISO 3015 [5] with a cooling rate of $-1{ }^{\circ} \mathrm{C} / \mathrm{min}$. An approximately $4 \mathrm{~mL}$ sample was placed in a cylindrical glass cup and heated on a plate heater set at $80{ }^{\circ} \mathrm{C}$ until fully melted prior to CP measurement. It should be noted that the effect of supercooling is often involved in CP measurement because the sample is cooled under an unagitated condition. Therefore, the precipitation behavior was also observed under ultrasonic treatment to mitigate the effect of supercooling. Approximately $10 \mathrm{~mL}$ of the sample was placed in a $50-\mathrm{mL}$ glass vial and fully melted on the plate heater. The sample was then cooled in an ultrasonic water bath (US-103, SND Co., Ltd., Nagano, Japan) shaking the vial by hand, and the temperature at which the solid phase first appeared was defined as the cloud point under 
the ultrasonic treatment (CPUS). The temperature of the sample was measured by a thermocouple and the cooling rate was around -10 to $-20{ }^{\circ} \mathrm{C} / \mathrm{min}$. The precipitation behavior was observed under storage at constant temperatures. Approximately $10 \mathrm{~mL}$ samples were placed in 50-mL glass vials, fully melted, and then held in a thermostatic chamber (FLI-301NH, Tokyo Rikakikai Co., LTD., Tokyo, Japan) set at constant temperatures from 0 to $30{ }^{\circ} \mathrm{C}$, and confirmed whether precipitates were formed once within 12 hours.

The precipitates formed during the above experiments were analyzed by X-ray diffraction (XRD, RINT2200V, Rigaku Corp., Tokyo, Japan). A portion of the precipitates were collected with a pipette, transferred into a capillary tube together with the liquid fraction and directly analyzed by XRD in transmission mode. The precipitates were also observed with the liquid fraction by a microscope (OPTIPHOT XF-21, Nikon Corp., Tokyo, Japan) through crossed polars.

Pure precipitates were isolated from the liquid fractions using centrifugation tubes with a $0.45 \mu \mathrm{m}$ membrane filter and the solid phases obtained were washed with a small amount of $n$-hexane. The pure precipitates obtained were analyzed by XRD in refraction mode to identify the crystalline structure. 


\section{Thermodynamic model}

The temperature of solid-liquid equilibrium ( $\left.T_{\mathrm{SLE}}\right)$ of 1-monopalmitin in methyl

oleate was thermodynamically calculated according to our another study using equation 1 [13].

$$
\exp \left[\left(\frac{T_{S L E}-T_{m, i}}{R T_{S L E}}\right)\left(\frac{\Delta H_{m, i}}{T_{m, i}}\right)\right]=\gamma_{i}^{L} x_{i}
$$

where $\gamma_{i}^{L}$ and $x_{\mathrm{i}}$ are activity coefficient and mole fraction of component $i$ in the liquid phase, respectively. The $\Delta H_{m, i}$ and $T_{m, i}$ are enthalpy of fusion and the melting point of pure component $i$, respectively. In this equation, we assumed that the solid phase that formed at $T_{\text {SLE }}$ is composed of only a single-component [13]. The modified UNIFAC (Dortmund) model was applied to calculate $\gamma_{i}^{L}$ and the measured values used for $\Delta H_{m, i}$ and $T_{m, i}$ were those from our another study [13].

\section{Results and Discussion}

Figure 1 shows the photographs of binary mixtures of $1 \mathrm{wt} \% 1$-monopalmitin in methyl oleate after CP measurements (a) without and (b) with ultrasonic treatment. A cloud of wax appeared in both cases owing to the precipitation formation. As mentioned 
later, X-ray diffraction analyses indicated that both precipitates obtained were composed mainly of 1-monopalmitin. Hence, these photographs show that MGs can make a cloud even it is low concentration.

Figure 2 shows the CPs of binary mixtures of 1-monopalmitin in methyl oleate compared with the calculated solid-liquid equilibrium temperatures ( $\left.T_{\text {SLE }}\right)$ of 1-monopalmitin. The measured CPs (CP, shown by open circles) are very close to the equilibrium curve for the $\alpha$ form of 1-monopalmitin. In spite of the very low concentrations of 1-monopalmitin, the CP is significantly affected by this MG because of its high melting point. The CPs measured under ultrasonic treatment (CPUs, shown by filled circles) are consistent with the equilibrium curve for the $\beta^{\prime}$ form. These results indicate that 1-monopalmitin is unlikely to crystallize as the $\beta$ form in methyl oleate, even if the temperature drops below the equilibrium temperature of the $\beta$ form. In the case under ultrasonic treatment, 1-monopalmitin seems to crystallize directly as the $\beta^{\prime}$ form immediately after the temperature reaches the equilibrium curve of the $\beta^{\prime}$ form without the effect of supercooling. Similar behaviors were also found in the case cooled with agitation by a magnetic stirrer in our another study [13]. However, 1-monopalmitin in methyl oleate may be supercooled under the unagitated condition of ordinary CP 
measurement. When the temperature was further decreased below the equilibrium temperature of the $\alpha$ form, 1-monopalmitin immediately crystallizes as the $\alpha$ form and this temperature is measured as the CP.

Figure 3 shows the times at which the formation of precipitates were first confirmed when the samples were stood at constant temperatures from 0 to $30{ }^{\circ} \mathrm{C}$. In the case of 1.0 wt $\%$ 1-monopalmitin in methyl oleate, precipitates formed within 1 hour when the storage temperature was lower than the $T_{\text {SLE }}$ of the $\alpha$ form, because the temperature was below the CP of the sample. When the storage temperature was higher than the $T_{\text {SLE }}$ of the $\alpha$ form, the precipitated time was abruptly increased and seems to be asymptotically closed to the $T_{\mathrm{SLE}}$ of the $\beta^{\prime}$ form. Similar behaviors were also observed in cases of $0.5 \mathrm{wt} \%$ (b) and $0.1 \mathrm{wt} \%$ (c). However, when the storage temperature was higher than the $T_{\text {SLE }}$ of the $\beta^{\prime}$ form, precipitates did not formed in any case as shown by cross marks. This indicates that 1-monopalmitin cannot crystallize as the $\beta$ form under storage at constant temperatures, similar to the results shown in Fig. 1. At temperatures lower than the $T_{\text {SLE }}$ of the $\beta^{\prime}$ form, 1-monopalmitin can directly crystallize as the $\beta^{\prime}$ form after a certain period under the constant temperature in an unagitated condition. 
Figure 4 shows the changes in the visual appearance for the mixture of $1 \mathrm{wt} \%$ 1-monopalmitin in methyl oleate held at $20^{\circ} \mathrm{C}$, which is higher than the $\mathrm{CP}$, for 14 days with their XRD patterns measured together with the liquid fraction in the transmission mode. The visual appearance of the precipitates did not appear to change much once solids had formed in the vial, even after 14 days. The intensity of the XRD pattern gradually increased with storage time, probably indicating the growth of 1-monopalmitin crystals. However, the diffraction patterns observed were too broad to identify its crystalline structure, probably because of the presence of the liquid phase.

Microscopic observations of the precipitates in the liquid fraction were conducted through crossed polars (Fig. 5), in which the white areas indicate the presence of crystals and black sections are from the liquid phase. Crystals formed in every case but their morphologies are quite different depending on the conditions under which the crystals formed. Crystals obtained under the conditions of ordinary CP measurement (a) were relatively large and flaky. In case of the CP measurement under ultrasonic treatment (b), agglomerated fine crystals formed. The crystals obtained when the mixture was held at $20^{\circ} \mathrm{C}$ (c) were rod-shaped. 
The crystallinity and form of the precipitates formed in the solutions were confirmed by XRD analyses conducted in refraction mode for the pure precipitates after the liquid fractions were completely removed. The XRD spectra of the precipitates are shown in Fig. 6, with standard samples of $\alpha$ (including sub- $\alpha$ ), $\beta^{\prime}$ and $\beta$ crystal forms of 1-monopalmitin for comparison. The XRD pattern of the precipitates obtained by the ordinary CP measurement (a) differs from that of the $\alpha$ form and is unexpectedly similar to that of the $\beta^{\prime}$ form. The precipitates obtained by CP measurement under ultrasonic treatment (b) and by standing at $20{ }^{\circ} \mathrm{C}$ (c) have XRD spectra that are consistent with the pattern of $\beta^{\prime}$ form. The precipitates of (b) and (c) crystallized as $\beta^{\prime}$ form in methyl oleate because precipitates formed at temperatures that were higher than the $T_{\text {SLE }}$ of the $\alpha$ form and the $\beta^{\prime}$ crystal have not converted to the $\beta$ form.

It is unclear why the precipitates obtained by the ordinary CP measurement (a) were the $\beta^{\prime}$ form when CP showed good agreement with the TSLE of the $\alpha$ form (Fig. 1). One possibility is that the CP matching the $T_{\mathrm{SLE}}$ of the $\alpha$ form is coincidental and the crystals are the $\beta^{\prime}$ form. In this case the deviation between the CP and $T_{\text {SLE }}$ of the $\beta^{\prime}$ form could be rationalized by the effect of supercooling. However, the experimental CP values were reproducible and the deviation between the CP and the TSLE of $\beta^{\prime}$ seems too large to 
be explained by the supercooling effect. We postulate that the CP is related with the formation of the $\alpha$ crystal form. Some possible explanations for the $\beta^{\prime}$ form in the XRD spectra are that the $\alpha$ type crystals form and are converted to the $\beta^{\prime}$ form during the experimental procedure; or that $\alpha$ type crystals form first and act as nuclei for $\beta^{\prime}$ crystal growth. However, further studies are required to elucidate the mechanism of precipitation at the CP.

\section{Conclusion}

$\mathrm{CP}$ measurements and the observation of precipitation at constant temperatures were conducted using mixtures of 1-monopalmitin and methyl oleate to understand the effect of MG polymorphism on the cold-flow properties of biodiesel. Precipitates formed in the mixtures at temperatures higher than the CPs when the mixtures were held at constant temperatures below the calculated $T_{\text {SLE }}$ of the $\beta^{\prime}$ form of 1-monopalmitin. The precipitates obtained were identified as the $\beta^{\prime}$ form of 1-monopalmitin by XRD analyses, proving that 1-monopalmitin can crystallize directly as the $\beta^{\prime}$ form in methyl oleate. The CPs of the mixtures were close to the $T_{\text {SLE }}$ of the $\alpha$ form of 1-monopalmitin, implying that the precipitation at the CP relates to the crystallization of the $\alpha$ form. However, the 
precipitates obtained at the CP had $\beta^{\prime}$ structure, and the mechanism of precipitation at the CP remains unclear.

The effect of MG polymorphism on precipitation was determined in this study, and the generally accepted concept of using the $\mathrm{CP}$ as an indicator of cold-flow fuel properties would not be appropriate when MGs affect the CP of biodiesel. The possibility of crystallization of MGs in the $\beta^{\prime}$ form needs to be considered. We also should study multi-component mixtures including real biodiesel to establish more practical estimation of cold-flow fuel properties.

Conflict of Interest: The authors declare that they have no conflict of interest. 


\section{References}

1. Haseeb ASMA, Fazal MA, Jahirul MI, Masjuki HH (2011) Compatibility of automotive materials in biodiesel: A review. Fuel 90:922-931

2. Moser BR (2009) Biodiesel production, properties, and feedstocks. Vitr Cell Dev Biol - Plant 45:229-266

3. Dunn RO, Bagby MO (1995) Low-temperature properties of triglyceride-based diesel fuels: Transesterified methyl esters and petroleum middle distillate/ester blends. J Am Oil Chem Soc 72:895-904

4. Lopes JCA, Boros L, Krähenbuühl MA, Meirelles AJA, Daridon JL, Pauly J, Marrucho IM, Coutinho JAP (2008) Prediction of cloud points of biodiesel. Energy Fuels 22:747-752

5. International Organization for Standarization (1992) Petroleum products -Determination of cloud point. ISO 3015:1992. 1-4

6. Tang H, De Guzman RC, Salley SO, Ng KYS (2008) Formation of insolubles in palm oil-, yellow grease-, and soybean oil-based biodiesel blends after cold soaking at 4 oC. J Am Oil Chem Soc 85:1173-1182 
7. Lutton ES, Jackson FL (1948) The Polymorphism of 1-Monostearin and 1-Monopalmitin. J Am Oil Chem Soc 70:2245-2249

8. Lutton ES (1950) Review of the Polymorphism of Saturated Even Glycerides. J Am Oil Chem Soc 27:276-281

9. Chapman D (1962) The Polymorphism of Glycerides. Chem Rev 62:433-456

10. Maruyama T, Niiya I, Imamura M, Okada M, Matsumoto T (1973) Study on Polymorphism of Monoglyceride . II. Thermodynamic Considerations on Transition. J Japan Oil Chem Soc 22:85-88(in Japanese)

11. Chupka GM, Yanowitz J, Chiu G, Alleman TL, Mccormick RL (2011) Effect of saturated monoglyceride polymorphism on low-temperature performance of biodiesel. Energy Fuels 25:398-405

12. Chupka GM, Fouts L, Lennon JA, Alleman TL, Daniels DA, Mccormick RL (2014) Saturated monoglyceride effects on low-temperature performance of biodiesel blends. Fuel Process Technol 118:302-309

13. Yoshidomi S, Sugami Y, Minami E, Shisa N, Hayashi H, Saka S (2017) Predicting solid-liquid equilibrium of fatty acid methyl ester and monoacylglycerol mixtures as biodiesel model fuels. J. Am. Oil Chem. Soc. 
14. Gmehling J, Li J, Schiller M (1993) A Modified UNIFAC Model. 2. Present Parameter Matrix and Results for Different Thermodynamic Properties. Ind Eng Chem Res 32:178-193 


\section{List of figures}

Fig.1 Photographs of binary mixtures of 1wt\% 1-monopalmitin in methyl oleate after CP measurements (a) without ultrasonic treatment and (b) with ultrasonic treatment.

Fig. 2 Cloud points of the binary mixtures of 1-monopalmitin in methyl oleate measured without agitation (CP) and measured under ultrasonic treatment (CPUS) with the calculated solid-liquid equilibrium temperatures (TSLE) for $\alpha, \beta^{\prime}$ and $\beta$ forms of 1-monopalmitin

Fig. 3 Precipitation times for the mixtures of (a) $1.0 \mathrm{wt} \%$, (b) $0.5 \mathrm{wt} \%$ and (c) $0.1 \mathrm{wt} \%$ 1-monopalmitin in methyl oleate at constant temperatures from 0 to $30^{\circ} \mathrm{C}$ in comparison with the $T_{\text {SLE }}$ of the $\alpha, \beta^{\prime}$ and $\beta$ forms (If precipitates were not observed, the finished times are shown by cross marks.)

Fig. 4 Changes in the visual appearance and XRD pattern measured in transmission mode

of the $1 \mathrm{wt} \% 1$-monopalmitin and methyl oleate mixture stored at $20{ }^{\circ} \mathrm{C}$ for 14 days 
Fig. 5 Microscopic observations viewed through crossed polars of the precipitates in the liquid fraction obtained by the ordinary CP measurement (a), the CP measurement under ultrasonic treatment (b) and by standing at $20{ }^{\circ} \mathrm{C}$ (c) for the mixture of $1 \mathrm{wt} \%$ 1-monopalmitin in methyl oleate

Fig. 6 XRD patterns of the precipitates obtained by the ordinary CP measurement (a), the $\mathrm{CP}$ measurement under ultrasonic treatment (b) and by standing at $20{ }^{\circ} \mathrm{C}$ (c) for the mixture of 1 wt\% 1-monopalmitin in methyl oleate. Standard samples of $\alpha+$ sub- $\alpha$, $\beta^{\prime}$ and $\beta$ crystals of 1 -monopalmitin shown for comparison 


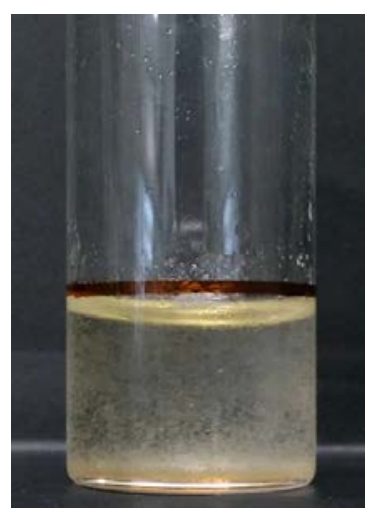

(a)

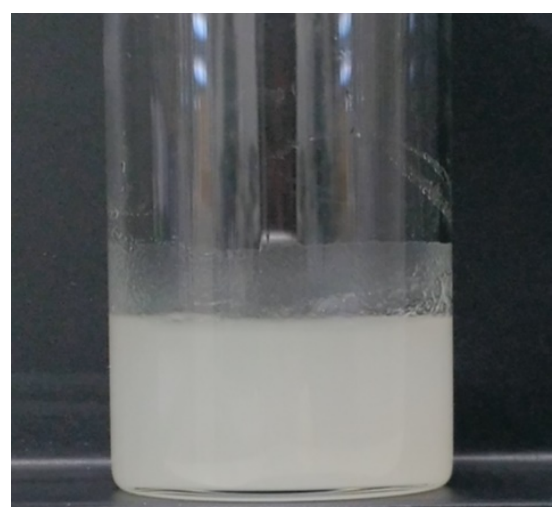

(b)

Fig. 1 


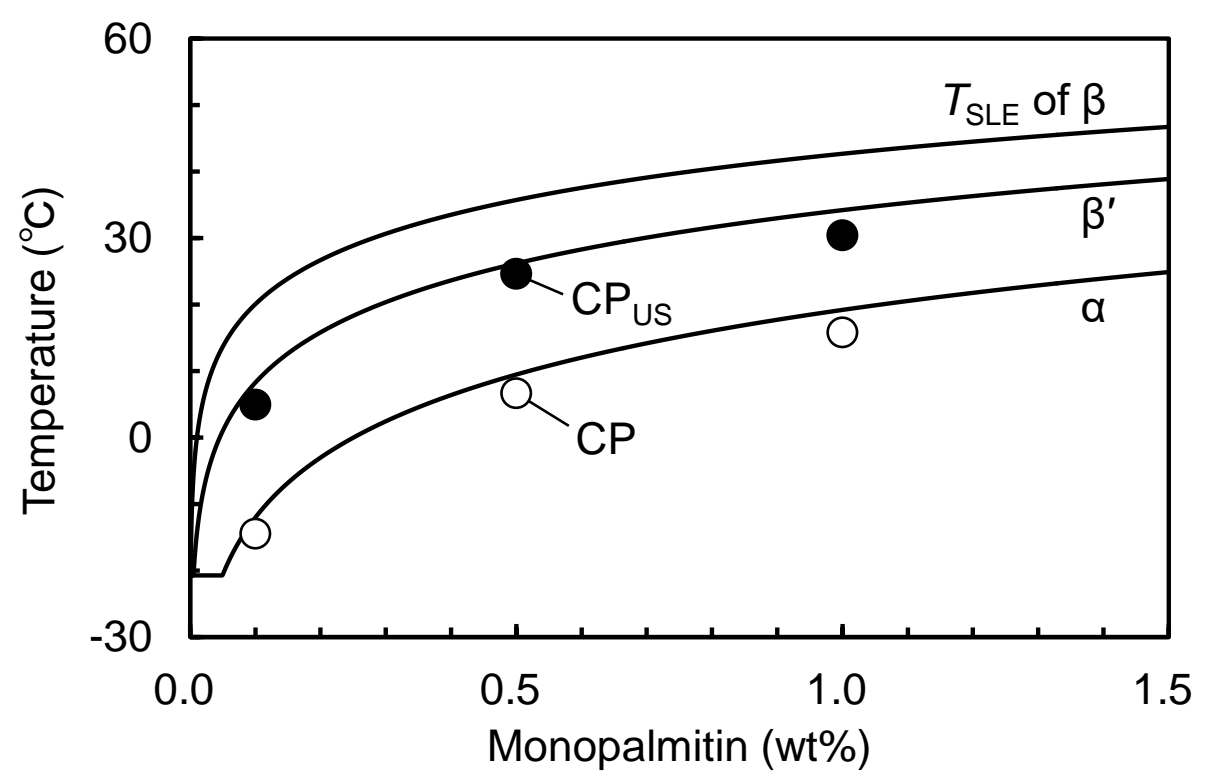

Fig. 2 
(a) $1.0 \mathrm{wt} \%$ of monopalmitin

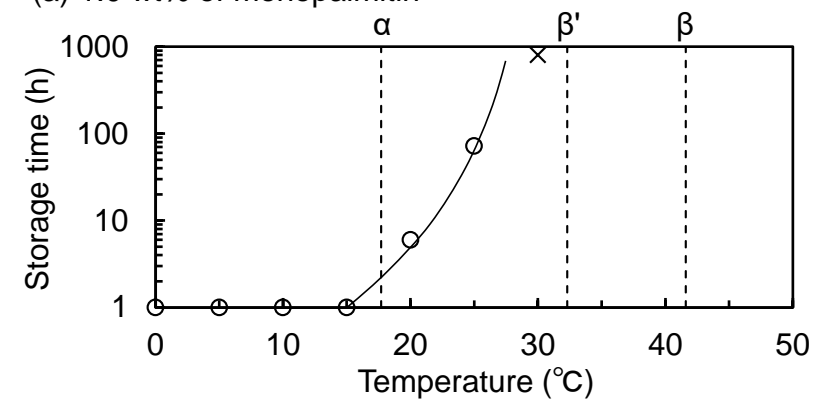

(b) $0.5 \mathrm{wt} \%$ of monopalmitin

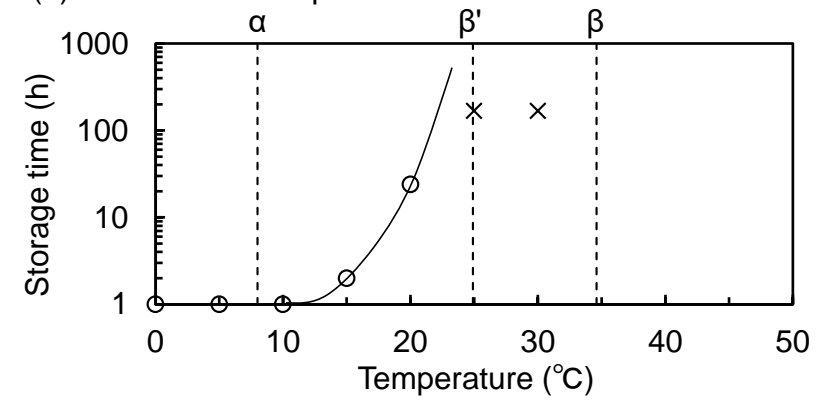

(c) 0.1 wt $\%$ of monopalmitin

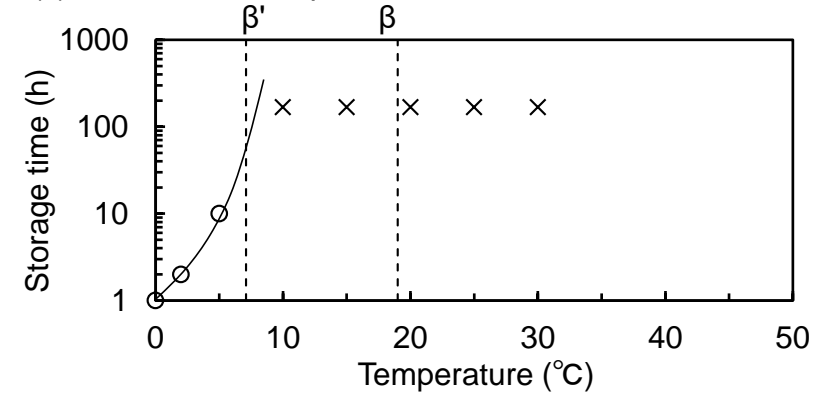

Fig. 3 

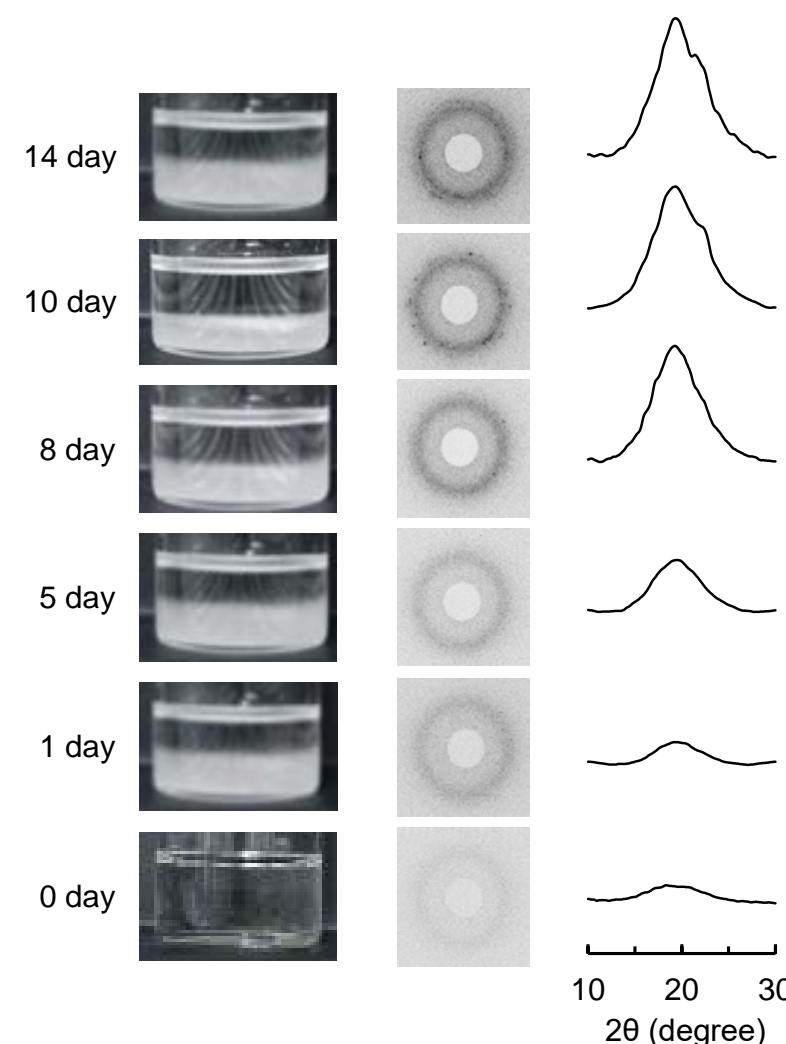

XRD pattern

Fig. 4 

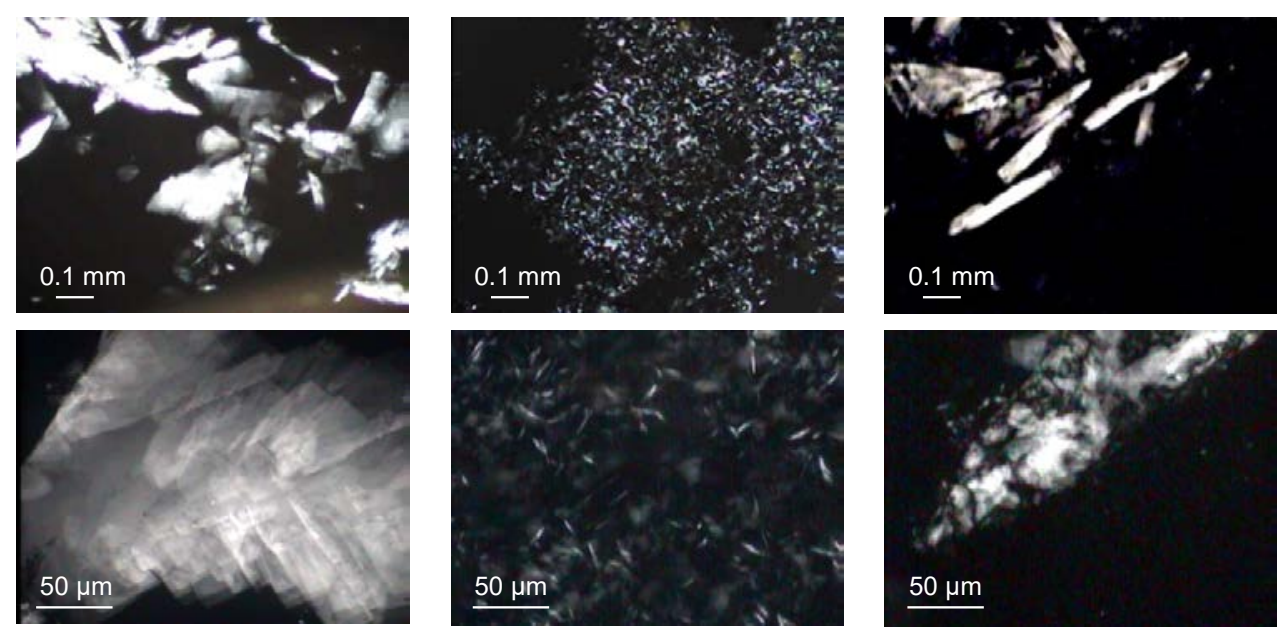

(a) $\mathrm{CP}$

(b) $\mathrm{CP}_{\text {US }}$

(c) at $20^{\circ} \mathrm{C}$

Fig. 5 


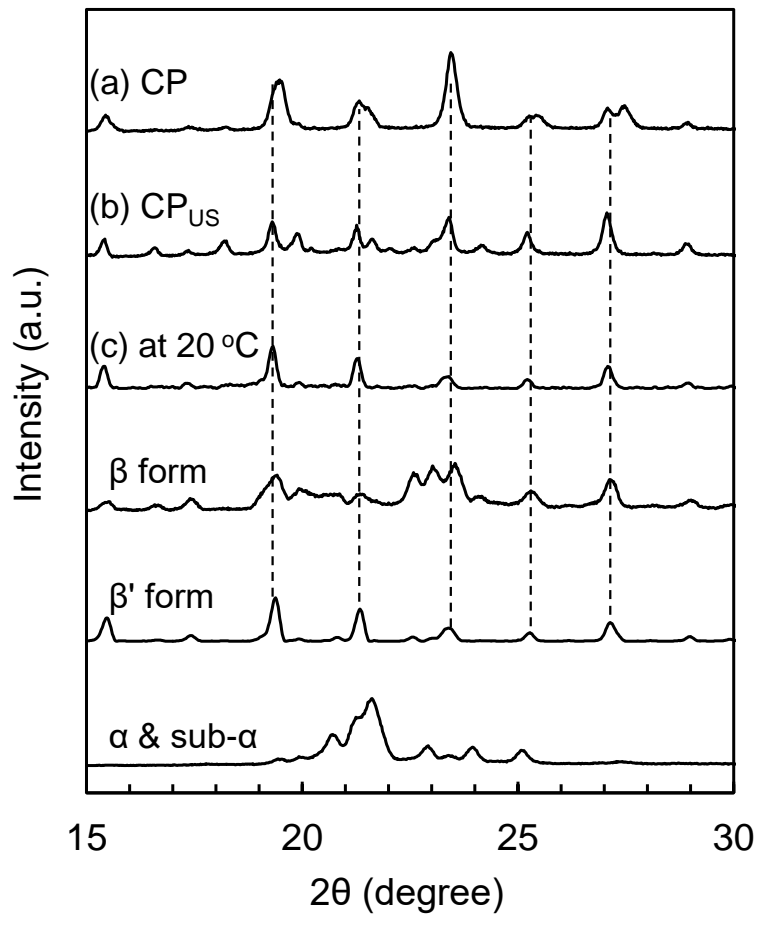

Fig. 6 